\title{
Comprehensiveness in the Research on Sustainability
}

\author{
Rajan Jose $^{1} \cdot$ Seeram Ramakrishna ${ }^{2}$
}

Published online: 3 January 2021

(C) The Author(s), under exclusive licence to Springer Nature Singapore Pte Ltd. part of Springer Nature 2021

\begin{abstract}
"Sustainability" has been defined as the "new normal" by many nations; many efforts are being undertaken for setting up a framework on "low-carbon economy" for sustainability. What does sustainability mean? Is it the maintenance of status quo on climate and resources? Or else, is it the reversal of existing conditions to the ones before the first industrial revolution? We would answer as "all these and beyond"; for example, eradication of poverty, creation of new jobs and resources for the growing population, and resilience from alienistic attack such as the novel corona virus are a few to mention to be included in the definition of sustainability. In our perspective, research on sustainability should have the following five components: resource sustainability, environmental protection, social well-being, knowledge integration, and circular economy, as schematically demonstrated in Fig. 1. The researcher's community has been investing substantial efforts on this topic; a simple Scopus search using the keyword "sustainability" on 14 Dec 2020 retrieved nearly 250,000 publications worldwide, which has grown from a single paper published in the year 1970 to nearly thirty thousand papers published in 2020 alone. Sustainability is therefore not a new topic for the learnt society and the knowledge enterprise; instead, it is a topic of steady momentum and popularity with time, which justifies our definition for sustainability.
\end{abstract}

Rajan Jose

rjose@ump.edu.my

Seeram Ramakrishna

seeram@nus.edu.m

1 Nanostructured Renewable Energy Materials Laboratory, Faculty of Industrial Sciences \& Technology, Universiti Malaysia Pahang, 26300 Kuantan, Malaysia

2 Center for Nanotechnology \& Sustainability, National University of Singapore, Singapore, Singapore
As of now, the sustainability research encompasses over a hundred keywords which range from sustainability of environment to secondary batteries, from life cycle analysis to energy efficiency, from resource sustainability to circular economy, and so on. In all sense, sustainability research is multidisciplinary; i.e., it addresses the real-life problem rather than mono-disciplines such as history, psychology, physics, chemistry, mathematics, or engineering. In the multidisciplinary approach, people from different disciplines work together, each drawing on their disciplinary knowledge, to create something better (Norström et al. 2020). Cross-disciplinary is viewing one discipline from the perspective of another. Other terminology often employed include interdisciplinary, which involves integrating knowledge and methods from different disciplines, thus meaning synthesis of approaches. Transdisciplinary involves creating a unity of intellectual frameworks beyond the disciplinary perspectives. All these research approaches are necessary for accelerating the sustainability solutions.

"Materials" occupy the largest share of economy at all levels of human life and could be classified into "materials for life sustenance" (e.g., food, food supplements, and medicines) and "materials for improving the living standards" (e.g., computers, bridges, vehicles, etc.). Obviously, the above classification defines sources of primary materials. "Materials for life sustenance" has directly or indirectly been resourced from plants and processed and can be grown and regrown. While sustainability is generally the case with plants, our habits ended up in the extinction of certain plant species (together with animal species) and some are nearing extinction. On the other hand, "materials for improving the living standards" are mined from the Earth, processed, value-added, and used for performing specific functions in devices and structures including life supporting machines. The terrestrial resources are anyway not renewable; the mines are not automatically filled up with similar resources with time. This is where the materials circular economy evolves: how do we reprocess an end used product as a resource of primary materials for making an improved product considering a growing population in mind? 


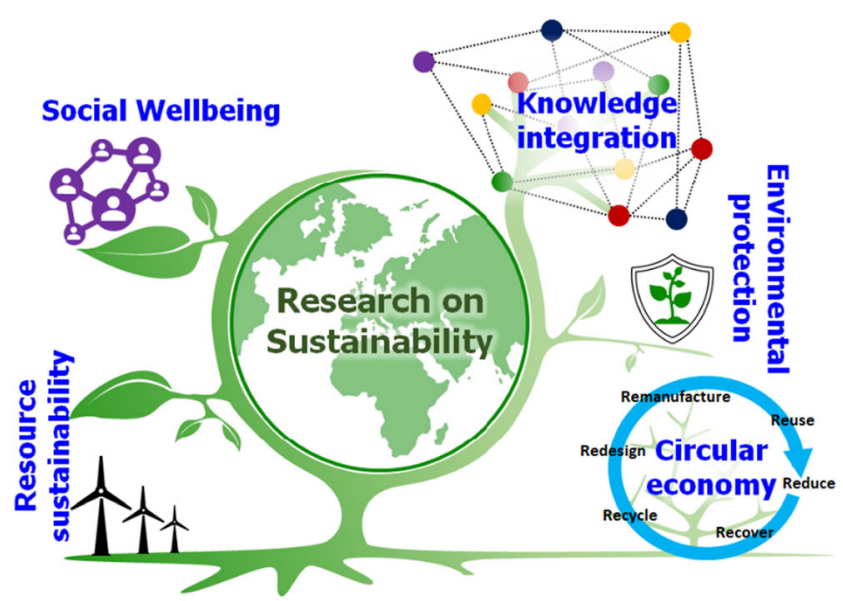

Fig. 1 Comprehensive sustainability research and its key pillars

How do we ensure that every atom in a used product is fed to manufacturing again? How do we ensure that the recycling process do not contribute adverse additives to the environment? That is, Materials Circular Economy is beyond just a recycling process but is fundamental to preserve the planet for the generations to come for their safer and comfortable habitat. Circular economy is projected to be the next major job creator (Stahel 2016), many realms of which are yet to be conceived. In the journal Materials Circular Economy, we pledge to highlight these important activities for a sustainable planet Earth and its beings.

\section{References}

Norström AV, Cvitanovic C, Löf MF et al (2020) Principles for knowledge co-production in sustainability research. Nat Sustain 3:182190. https://doi.org/10.1038/s41893-019-0448-2

Stahel WR (2016) The circular economy. Nature 531:435-438. https:// doi.org/10.1038/531435a 